Case Report

\title{
A Case of Aspiration Pneumonia Caused by Cerebrospinal Fluid Leaks Associated with Delayed Identification of Iatrogenic Skull Base Injury during Endoscopic Sinus Surgery
}

\author{
Takashi Anzai $\left(\mathbb{D},{ }^{1}\right.$ Akira Baba, ${ }^{2}$ Shin Ito, ${ }^{1}$ Yo Suzuki, ${ }^{1}$ Shori Tajima ${ }^{1 D},{ }^{1}$ Satoshi Hara, \\ Yusuke Takata, ${ }^{1}$ Fumihiko Matsumoto, ${ }^{1}$ and Katsuhisa Ikeda ${ }^{1}$ \\ ${ }^{1}$ Department of Otorhinolaryngology, Juntendo University Faculty of Medicine, Tokyo, Japan \\ ${ }^{2}$ Department of Radiology, The Jikei University School of Medicine, Tokyo, Japan \\ Correspondence should be addressed to Takashi Anzai; tanzai@juntendo.ac.jp
}

Received 16 February 2021; Revised 23 March 2021; Accepted 29 March 2021; Published 9 April 2021

Academic Editor: Toshihisa Murofushi

Copyright (C) 2021 Takashi Anzai et al. This is an open access article distributed under the Creative Commons Attribution License, which permits unrestricted use, distribution, and reproduction in any medium, provided the original work is properly cited.

\begin{abstract}
Cerebrospinal fluid (CSF) leaks associated with endoscopic sinus surgery (ESS) are a rare complication affecting approximately $0.09 \%$ of patients. Although meningitis is a well-known complication of CSF leaks, the case we present is a rare and cautionary case of CSF leakage associated with ESS leading to aspiration pneumonia. A 43-year-old man with CSF leaks after ESS was referred to our hospital. After the operation, sometimes, he reported having a serous nasal discharge from the right side when he bent over, and he woke up choking on something every day. He also experienced headache, fever, fatigue, and cough. Interestingly, chest computed tomography (CT) showed a consolidation and ground-glass opacity in the posterior segments of the right upper lobes and superior segments of the bilateral lower lobes. These CT imaging findings were similar to those of aspiration pneumonia in bedridden patients who are always in a supine position. These findings suggest that CSF caused aspiration pneumonia. To the best of our knowledge, no case of aspiration pneumonia caused by CSF during endoscopic sinus surgery has been reported until now. If a patient with CSF leakage after ESS experiences fever, cough, or fatigue, physicians should consider aspiration pneumonia in addition to meningitis.
\end{abstract}

\section{Introduction}

Cerebrospinal fluid (CSF) leak associated with endoscopic sinus surgery (ESS) is a rare complication affecting approximately $0.09 \%$ of patients [1]. If the surgeon is immediately aware that the skull base has been injured, he or she can take the necessary action at that time. However, in rare cases, the surgeon may not realize that the skull base has been injured during surgery. In such cases, the presence of CSF leaks becomes evident only when the patient complains of a serous nasal discharge, headache, or fever after surgery. Although meningitis is a well-known complication of CSF leaks, the case we present is a rare and cautionary case of CSF leaks associated with ESS leading to aspiration pneumonia.

\section{Case Report}

A 43-year-old man with CSF leaks after ESS was referred to the Department of Otolaryngology at Juntendo University Hospital by a surgical clinic. His medical history was unremarkable. He underwent right-side ESS 9 days before and left-side ESS 6 days before presenting to our hospital. His right-side nasal packing was removed at the time of the left-side operation. After the operation on the left, sometimes, he reported having a serous nasal discharge from the right side when he bent over, and he woke up choking on something every day. He also experienced headache, fever, fatigue, and cough. He returned to the surgical clinic and was diagnosed with CSF leakage from his right skull base. At that time, nasal packings were inserted in the right side, and the CSF leakage was stopped temporarily. 


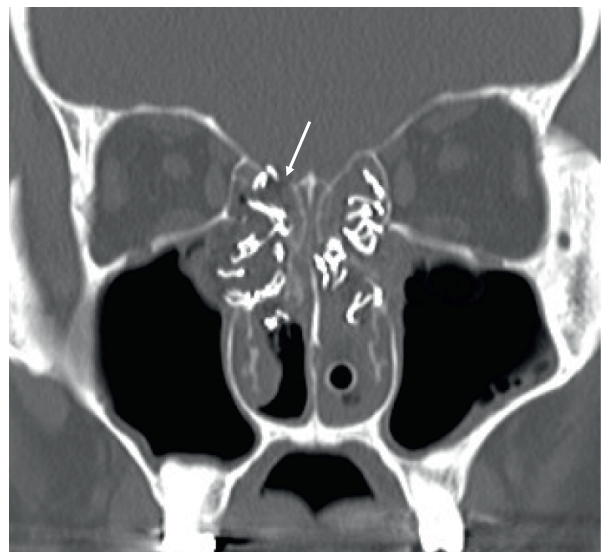

FIgURE 1: The head and neck computed tomography (CT) radiological findings. The head and neck coronal CT in the bone window setting revealed a defect in the cribriform plate (arrow), consistent with a cerebrospinal fluid leak.

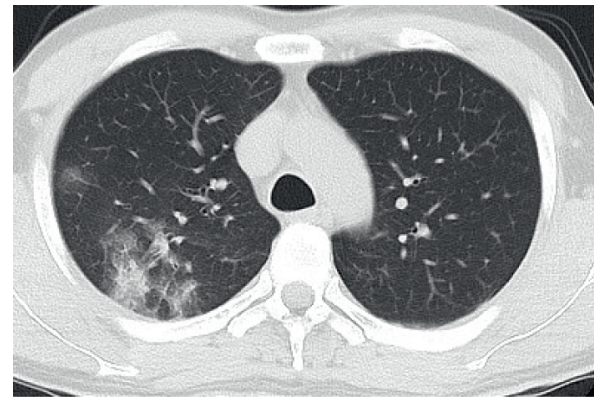

(a)

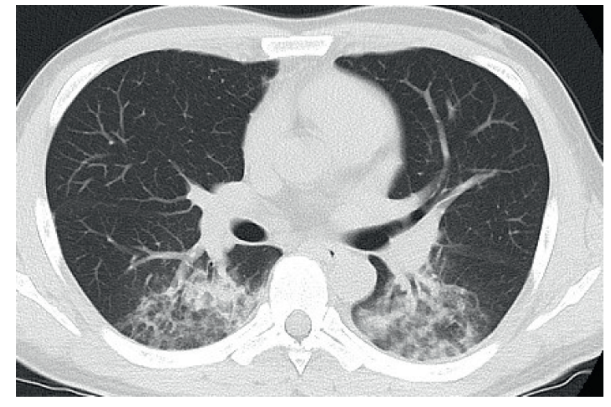

(b)

FIgURE 2: Preoperative chest computed tomography (CT) radiological findings. A chest axial CT in the lung window setting showed a consolidation and ground-glass opacity in the posterior segments of the right upper lobes (a) and the superior segments of the bilateral lower lobes (b), consistent with aspiration pneumonia.

$\mathrm{He}$ was then transferred to our hospital. $\mathrm{His} \mathrm{SpO}_{2}$ was $98 \%$ on room air, and his body temperature was $37.4^{\circ} \mathrm{C}$. He reported having a headache, but neck stiffness was not observed. His bilateral nasal cavities were packed with gauzes. Postnasal discharge was not detected at his retropharyngeal wall. Blood test results suggested inflammation (white blood cell count: $11.6 \times 10^{9} / \mathrm{L}$; C-reactive protein: $12.63 \mathrm{mg} / \mathrm{L}$ ). A CSF test showed no evidence of meningitis and had a clear appearance (microscopy: white blood cell, $0 /$ $\mathrm{mm}^{3}$; red blood cell, $0 / \mathrm{mm}^{3}$; protein, $0.18 \mathrm{~g} / \mathrm{L}$; CSF glucose, $5.4 \mathrm{mmol} / \mathrm{L}$ ). Computed tomography (CT) scans showed a defect in the cribriform plate (Figure 1).

Because he had fever, cough, and fatigue, we performed chest CT and a severe acute respiratory syndrome coronavirus-2 polymerase chain reaction test to screen for coronavirus disease 2019. The polymerase chain reaction test was performed twice and was negative both times. Interestingly, chest CT showed a consolidation and ground-glass opacity in posterior segments of the right upper lobes and superior segments of the bilateral lower lobes (Figure 2), indicating aspiration pneumonia. A sputum culture test showed a negative result.

We performed endoscopic transnasal CSF leak repair under general anesthesia. The bone defect and CSF leakage were found in the right frontal ethmoidal skull base
(Figure 3(a)). Two pieces of temporal muscle fascia were harvested. One piece of fascia was introduced through the dural defect into the intracranial cavity. Then, the graft was gently pulled with forceps to plug the fistulae (Figure 3(b)). Another piece of fascia was placed as the overlay graft as the second layer (Figure 3(c)). Finally, the septal mucosal flap was placed as the third layer (Figure 3(d)). These grafts were fixed with fibrin glue.

After this operation, the CSF leaks had stopped, the patient's temperature returned to normal, and the chest CT scan showed normal findings (Figure 4).

\section{Discussion}

Aspiration pneumonia occurs after the aspiration of microorganisms from the oral cavity or nasopharynx. It has been reported that the posterior segments of the upper lobes and superior segments of the lower lobes are typically involved in supine patients and that the posterior segments of the lower lobes are involved in upright patients $[2,3]$. The case we report here with CSF leaks associated with iatrogenic skull base injury during ESS had developed pneumonia. The pneumonia was concentrated in the posterior segments of the right upper lobes and the superior segments of the bilateral lower lobes. Interestingly, the chest CT showed 


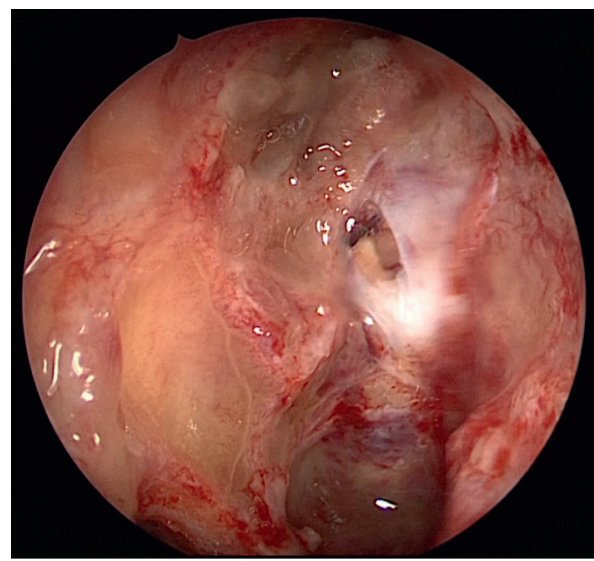

(a)

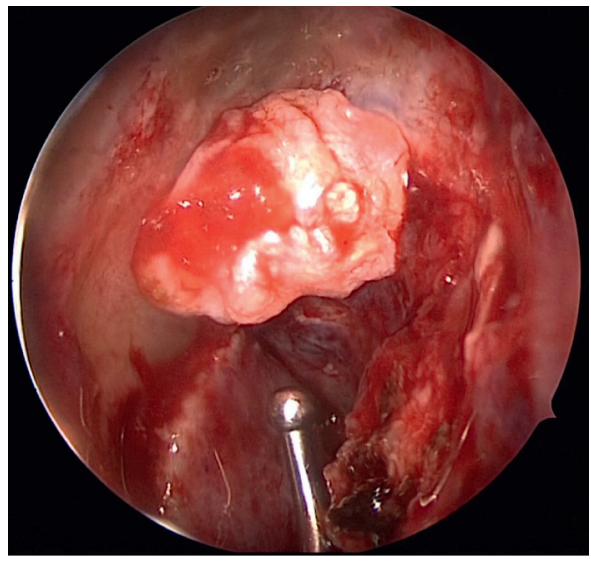

(c)

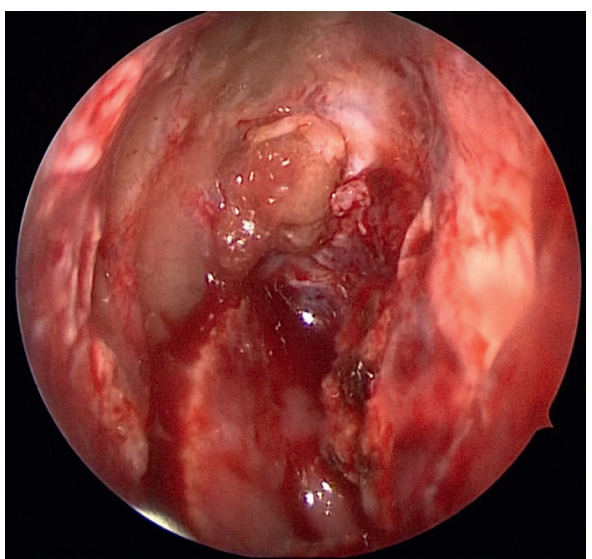

(b)

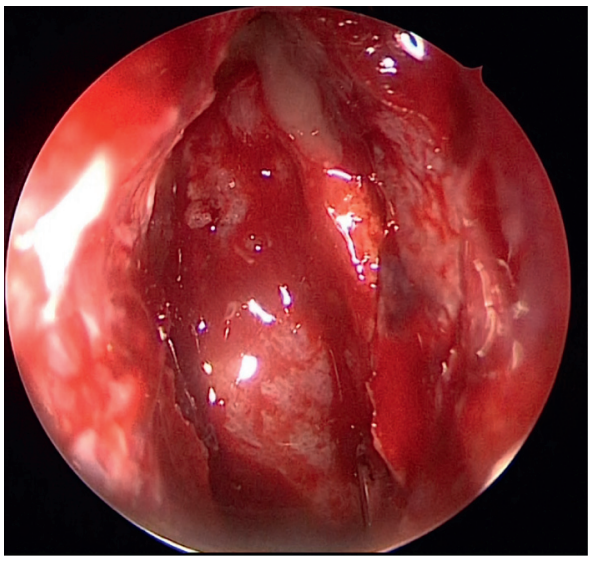

(d)

FIgURE 3: Intraoperative findings. (a) The bone defect and cerebrospinal fluid leaks were found in the right frontal ethmoidal skull base. (b) A piece of fascia was introduced through the dural defect into the intracranial cavity. The graft was gently pulled with forceps to plug the fistulae. (c) Another piece of fascia was placed as an overlay graft as the second layer. (d) A septal mucosal flap was placed as the third layer.

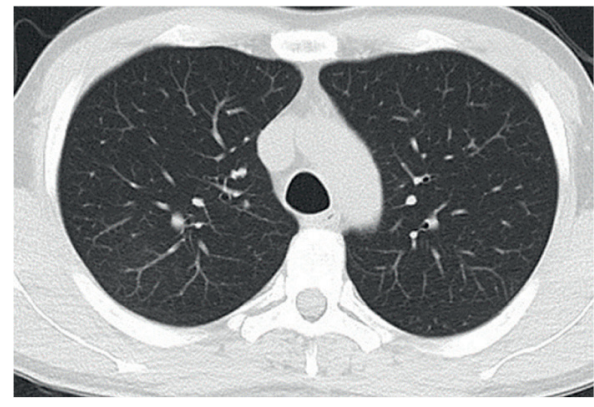

(a)

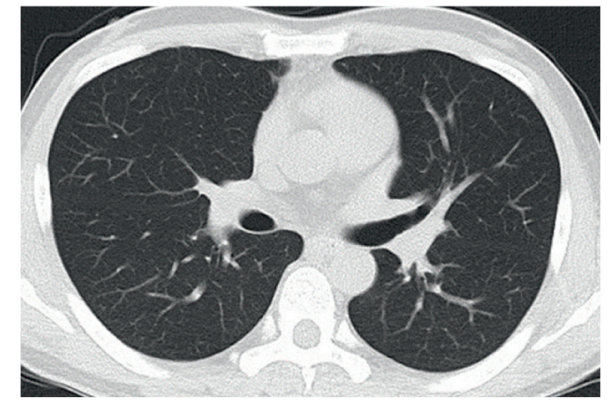

(b)

Figure 4: Postoperative chest computed tomography (CT) radiological findings. A chest CT in the lung window setting showed no consolidation or ground-glass opacity.

imaging findings similar to those of aspiration pneumonia in bedridden patients who are always in a supine position. It was recently reported that spontaneous CSF leaks can cause chronic aspiration pneumonitis $[4,5]$. The pathology in this case may have had the same mechanism. The patient was a healthy adult with no functional swallowing problem, but woke up choking on CSF postnasal discharge every night after the right nasal packing was removed. Frequent aspiration of the spinal fluid into the larynx during unconsciousness may have resulted in aspiration pneumonia. In the present case, the CSF leak may have caused aspiration pneumonia because there was a relatively large amount of CSF leakage owing to a large skull base and dural defect and because it took a relatively long time to detect the CSF 
leakage. If a patient with CSF leak after ESS experiences fever, cough, or fatigue, physicians should consider aspiration pneumonia in addition to meningitis.

\section{Conclusion}

The chest CT in the case of CSF leaks associated with delayed identification of iatrogenic skull base injury during endoscopic sinus surgery showed imaging findings similar to those of aspiration pneumonia found in bedridden patients who are always in a supine position.

If a patient with CSF leak after ESS experiences fever, cough, or fatigue, physicians should consider aspiration pneumonia in addition to meningitis.

\section{Ethical Approval}

This case report was written in accordance with the Declaration of Helsinki.

\section{Conflicts of Interest}

The authors declare no conflicts of interest associated with this manuscript.

\section{Authors' Contributions}

TA and AB prepared and edited this manuscript. SI, YS, ST, $\mathrm{SH}, \mathrm{YT}, \mathrm{FM}$, and IK contributed to the collection of data. $\mathrm{TA}, \mathrm{AB}$, and $\mathrm{KI}$ gave the final approval for this version of the manuscript. All authors read and approved the final manuscript.

\section{Acknowledgments}

This work was supported in part by the Japan Society for the Promotion of Science KAKENHI (Grant no. 20K09721 to S Ito).

\section{References}

[1] M. Koizumi, S. Suzuki, H. Matsui, K. Fushimi, T. Yamasoba, and H. Yasunaga, "Trends in complications after functional endoscopic sinus surgery in Japan: a comparison with a previous study (2007-2013 vs. 2013-2017)," Auris Nasus Larynx, vol. 47, pp. 814-819, 2020.

[2] A. D. Prather, T. R. Smith, D. M. Poletto et al., "Aspirationrelated lung diseases," Journal of Thoracic Imaging, vol. 29, pp. 304-309, 2014.

[3] L. A. Mandell and M. S. Niederman, "Aspiration pneumonia," The New England Journal of Medicine, vol. 380, no. 7, pp. 651-663, 2019.

[4] M. Or, I. A. Buchanan, S. Sizdahkhani et al., "Chronic aspiration pneumonitis caused by spontaneous cerebrospinal fluid fistulae of the skull base," The Laryngoscope, 2020.

[5] J. Seltzer, A. Babadjouni, B. B. Wrobel, and G. Zada, "Resolution of chronic aspiration pneumonitis following endoscopic endonasal repair of spontaneous cerebrospinal fluid fistula of the skull base," Journal of Neurological Surgery Reports, vol. 77, pp. 73-76, 2016. 